\title{
Proper actions of locally compact groups on equivariant absolute extensors
}

\author{
by
}

Sergey Antonyan (México)

\begin{abstract}
Let $G$ be a locally compact Hausdorff group. We study equivariant absolute (neighborhood) extensors ( $G$-AE's and $G$-ANE's) in the category $G$ - $\mathcal{M}$ of all proper $G$-spaces that are metrizable by a $G$-invariant metric. We first solve the linearization problem for proper group actions by proving that each $X \in G-\mathcal{M}$ admits an equivariant embedding in a Banach $G$-space $L$ such that $L \backslash\{0\}$ is a proper $G$-space and $L \backslash\{0\} \in G$-AE. This implies that in $G-\mathcal{M}$ the notions of $G-\mathrm{A}(\mathrm{N}) \mathrm{E}$ and $G-\mathrm{A}(\mathrm{N}) \mathrm{R}$ coincide. Our embedding result is applied to prove that if a $G$-space $X$ is a $G$-ANE (resp., a $G$-AE) such that all the orbits in $X$ are metrizable, then the orbit space $X / G$ is an ANE (resp., an AE if, in addition, $G$ is almost connected). Furthermore, we prove that if $X \in G-\mathcal{M}$ then for any closed embedding $X / G \hookrightarrow B$ in a metrizable space $B$, there exists a closed $G$-embedding $X \hookrightarrow Z$ (a lifting) in a $G$-space $Z \in G$ - $\mathcal{M}$ such that $Z / G$ is a neighborhood of $X / G$ (resp., $Z / G=B$ whenever $G$ is almost connected). If a proper $G$-space $X$ has metrizable orbits and a metrizable orbit space then it is metrizable (by a $G$-invariant metric).
\end{abstract}

1. Introduction. In order to extend the theory of compact transformation groups to locally compact ones, in $1961 \mathrm{R}$. Palais [42] introduced the fundamental concept of a proper action of an arbitrary locally compact group $G$ on a completely regular Hausdorff space $X$. Among other important results he established, under the assumption that $G$ is a Lie group, the remarkable fact of existence of a slice at every point $x \in X$; this means that the orbit $G(x)$ is a $G$-equivariant neighborhood retract of $X$. In general, when $G$ is not a Lie group, it is no longer true that a slice exists at each point of $X$ (see [7]). However, for arbitrary locally compact (non-Lie) groups, an approximate version of Palais' slice theorem holds true [14, Theorem 3.5].

We recall that an action of a locally compact Hausdorff group $G$ on a completely regular Hausdorff space $X$ is said to be proper [42, Definition 1.2.2] if every point $x \in X$ has a neighborhood $V_{x}$ such that for any point

2010 Mathematics Subject Classification: 57S99, 54C55, 54H15.

$K e y$ words and phrases: proper $G$-space, $G$-AE, equivariant embedding, orbit space, approximate slice. 
$y \in X$ there exists a neighborhood $V_{y}$ with the property that the set $\left\langle V_{x}, V_{y}\right\rangle=$ $\left\{g \in G \mid g V_{x} \cap V_{y} \neq \emptyset\right\}$ has compact closure in $G$. In this case $X$ is called a proper $G$-space. Clearly, if $G$ is compact, then every $G$-space is proper.

In this paper, in particular, we are interested in extending some fundamental results of the equivariant theory of retracts from the case of compact group actions to the case of proper actions of arbitrary locally compact groups.

The passage to the locally compact (non-Lie) case is nontrivial and is based on the above mentioned approximate slice theorem from [14]. It turns out that for purposes of the equivariant theory of retracts the approximate slice theorem is needed rather than the "exact" slice theorem.

Based on this theorem, we first establish in Section 3 two equivariant embedding results which solve the linearization problem for proper actions of locally compact groups. Namely, Theorem 3.1 states that every invariantly metrizable proper $G$-space $X$ embedds equivariantly into a Banach $G$-space $L$ such that $L \backslash\{0\}$ is a proper $G$-space and a $G$-AE. This embedding, in general, is not closed. However, Theorem 3.2 states that $X$ admits an equivariant closed embedding in a proper $G$-AE space of the form $E \backslash D$, where $D$ is an invariant closed linear subspace of a normed linear $G$-space $E$, and $D$ is a $Z$-set in $E$ (see Remark 3.3). An immediate corollary is that an invariantly metrizable proper $G$-space is a $G$-A(N)R iff it is a $G-\mathrm{A}(\mathrm{N}) \mathrm{E}$ (see Corollary 3.10).

The first embedding results for proper $G$-spaces are due to Palais [42]. He proved that if $G$ is a matrix group and $X$ a finite-dimensional separable metrizable proper $G$-space having only finitely many orbit types, then $X$ admits a $G$-equivariant embedding in a linear $G$-space $L$ of finite dimension. In the same paper of Palais it is proved that if $G$ is any Lie group and $X$ a separable metrizable proper $G$-space, then $X$ admits a $G$-equivariant embedding in a real Hilbert $G$-space $L$, where the action is by means of linear orthogonal operators. However, in his constructions Palais did not care about the proper part of the ambient $G$-space $L$. In [24] E. Elfving improved this result of Palais by proving that if, in addition, $G$ is a linear Lie group and $X$ is locally compact, then the relevant $G$-embedding $X \hookrightarrow L$ may be arranged to be closed and such that the $G$-action is proper on some invariant neighborhood of $X$ in $L$.

In Section 7 we prove Theorem 7.1 which is the most general (in fact, final) result about preservation of equivariant extension properties by the orbit space functor. Its particular case (Corollary 7.2) states that if $G$ is a locally compact group and $X$ any $G$-ANE in which all the orbits are metrizable, then the orbit space $X / G$ is an ordinary ANE. If, in addition, $G$ is almost connected and $X$ a $G$-AE, then $X / G$ is an AE. The very first result of this sort (see Theorem 2.6 below) was established in [6] for actions of compact metrizable groups on metrizable spaces. Recently it was widely 
applied in the study of the topology of Banach-Mazur compacta (see [9], [11] and [13]). Other applications can be found in [6], [18] and [45].

The proof of Theorem 7.1 is based on the results of Sections 4-6 which are also of independent interest. In Section 4 we first prove Theorem 4.1, a particular case of Theorem 7.1 when $X$ is an invariantly metrizable proper $G$-space. Based on this result, in Section 5 we prove Theorem 5.1, devoted to equivariant liftings of closed embeddings. Its particular case states that if $G$ is a locally compact (resp., almost connected) group and $X$ an invariantly metrizable proper $G$-space, then for every closed embedding $X / G \hookrightarrow B$ in a metrizable space $B$, there exists an equivariant closed embedding $X \hookrightarrow Z$ (a lifting of $X / G \hookrightarrow B$ ) in an invariantly metrizable proper $G$-space $Z$ such that $Z / G$ is a neighborhood of $X / G$ in $B$ (resp., $Z / G=B$ ). This result is an important component of the proof of Theorem 7.1.

Theorem 6.1 of Section 6 , which is another ingredient of the proof of Theorem 7.1, generalizes a well known result of B. A. Pasynkov [43, Theorem 2] and V. V. Filippov [28, Theorem] to the case of noncompact group actions. It asserts that if $G$ is a locally compact group, then each proper $G$-space with metrizable orbits and metrizable orbit space is necessarily metrizable (by a $G$-invariant metric).

In Section 8 we prove some auxiliary algebraic results about almost connected groups and their maximal compact subgroups which we have used in the preceding sections.

2. Preliminaries. Throughout the paper the letter $G$ will denote a locally compact Hausdorff group unless otherwise stated; by $e$ we shall denote the unity of $G$.

All topological spaces and topological groups are assumed to be completely regular and Hausdorff. The basic ideas and facts of the theory of $G$-spaces and topological transformation groups can be found in Bredon [21] and in Palais [41]. Our basic references on proper group actions are Palais [42], Koszul [37] and Abels [1], [2]. For the equivariant theory of retracts the reader can see, for instance, [4]-[6] and [14].

For the convenience of the reader we recall, however, some definitions and facts.

By a $G$-space we mean a topological space $X$ together with a fixed continuous action $G \times X \rightarrow X$ of a topological group $G$ on $X$. By $g x$ we denote the image of the pair $(g, x) \in G \times X$ under the action.

If $Y$ is another $G$-space, a continuous map $f: X \rightarrow Y$ is called a $G$-map or an equivariant map if $f(g x)=g f(x)$ for every $x \in X$ and $g \in G$. If $G$ acts trivially on $Y$ then we use the term "invariant map".

By a normed linear $G$-space (resp., a Banach $G$-space) we mean a $G$ space $L$, where $L$ is a normed linear space (resp., a Banach space) on which 
$G$ acts by means of linear isometries, i.e., $g(\lambda x+\mu y)=\lambda(g x)+\mu(g y)$ and $\|g x\|=\|x\|$ for all $g \in G, x, y \in L$ and $\lambda, \mu \in \mathbb{R}$.

If $X$ is a $G$-space and $H$ a subgroup of $G$ then, for a subset $S \subset X$, $H(S)$ denotes the $H$-saturation of $S$, i.e., $H(S)=\{h s \mid h \in H, s \in S\}$. In particular, $H(x)$ denotes the $H$-orbit $\{h x \in X \mid h \in H\}$ of $x$. The quotient space of all $H$-orbits is called the $H$-orbit space and denoted by $X / H$.

If $H(S)=S$, then $S$ is said to be an $H$-invariant set. A $G$-invariant set will simply be called an invariant set.

For a closed subgroup $H \subset G$, we will denote by $G / H$ the $G$-space of cosets $\{g H \mid g \in G\}$ under the action induced by left translations.

If $X$ is a $G$-space and $H$ a closed normal subgroup of $G$, then the $H$ orbit space $X / H$ will always be regarded as a $G / H$-space endowed with the following action of $G / H:(g H) * H(x)=H(g x)$ for $g H \in G / H$ and $H(x) \in X / H$.

For any $x \in X$, the subgroup $G_{x}=\{g \in G \mid g x=x\}$ is called the stabilizer (or stationary subgroup) at $x$. For a subgroup $H \subset G$, the set $X^{H}=\left\{x \in X \mid H \subset G_{x}\right\}$ is called the $H$-fixed point set of $X$.

A compatible metric $\rho$ on a $G$-space $X$ is called invariant or $G$-invariant if $\rho(g x, g y)=\rho(x, y)$ for all $g \in G$ and $x, y \in X$.

A locally compact group $G$ is called almost connected if the space of connected components of $G$ is compact. Such a group has a maximal compact subgroup $K$, i.e., every compact subgroup of $G$ is conjugate to a subgroup of $K$ [1, Theorem A.5]. The corresponding classical theorem on Lie groups can be found in [30, Ch. XV, Theorem 3.1].

Let $X$ be a $G$-space. Two subsets $U$ and $V$ in $X$ are called thin relative to each other [42, Definition 1.1.1] if the set $\langle U, V\rangle=\{g \in G \mid g U \cap V \neq \emptyset\}$ (called the transporter from $U$ to $V$ ) has compact closure in $G$. A subset $U$ of a $G$-space $X$ is called small if every point in $X$ has a neighborhood thin relative to $U$. A $G$-space $X$ is called proper (in the sense of Palais) if every point in $X$ has a small neighborhood.

Clearly, if $G$ is compact, then every $G$-space is proper. Furthermore, if $G$ acts properly on a compact space, then $G$ has to be compact as well. If $G$ is discrete and $X$ is locally compact, the notion of a proper action is the same as the classical notion of a properly discontinuous action. When $G=\mathbb{R}$, the additive group of the reals, proper $G$-spaces are precisely the dispersive dynamical systems with regular orbit space (see [20, Ch. IV]).

Each orbit in a proper $G$-space is closed, and each stabilizer is compact [42, Proposition 1.1.4]. It is easy to check the following two statements: (1) the product of two $G$-spaces is proper whenever one of them is; (2) the inverse image of a proper $G$-space under a $G$-map is again a proper $G$-space. 
Important examples of proper $G$-spaces are the coset spaces $G / H$ with $H$ a compact subgroup of a locally compact group $G$. Other interesting examples can be found in [1], [2], [9], [24], [25], [33] and [37].

In the present paper we are especially interested in the class $G-\mathcal{M}$ of all metrizable proper $G$-spaces that admit a compatible $G$-invariant metric; we call them invariantly metrizable $G$-spaces. It is well-known that, for $G$ a compact group, the class $G-\mathcal{M}$ coincides with the class of all metrizable $G$-spaces (see [41, Proposition 1.1.12]). A fundamental result of R. Palais [42, Theorem 4.3.4] states that if $G$ is a Lie group, then $G-\mathcal{M}$ includes all separable metrizable proper $G$-spaces. The question of whether the separability can be omitted in this result of Palais still remains open (even for $G=\mathbb{R}$ and $G=\mathbb{Z}$ ). We refer to [17] for a further discussion of this interesting problem.

Another important subclass of $G-\mathcal{M}$ is formed by the twisted products $G \times_{K} S$, where $G$ is a locally compact metrizable group, $K$ a compact subgroup of $G$, and $S$ a metrizable $K$-space (see [12, Lemma 1.1]). It is known from [1, Main Theorem] that if $G$ is almost connected, then each $X \in G-\mathcal{M}$ has the form $G \times_{K} S$, where $K$ is a maximal compact subgroup of $G$, and $S$ is a $K$-space.

Let us recall that if $K$ is a closed subgroup of $G$, and $S$ is a $K$-space, then $G \times{ }_{K} S$ is the orbit space of the $K$-space $G \times S$ on which $K$ acts by $k(g, s)=\left(g k^{-1}, k s\right)$. Furthermore, there is a natural action of $G$ on $G \times{ }_{K} S$ given by $g^{\prime}[g, s]=\left[g^{\prime} g, s\right]$, where $g^{\prime} \in G$ and $[g, s]$ denotes the $K$-orbit of $(g, s)$ in $G \times S$. The twisted products are of particular interest in the theory of transformation groups (see [21, Ch. II, § 2]).

A $G$-space $Y$ is called an equivariant neighborhood extensor for a given $G$-space $X$ (notation: $Y \in G$-ANE $(X)$ ) if, for any closed invariant subset $A \subset X$ and any $G$-map $f: A \rightarrow Y$, there exist an invariant neighborhood $U$ of $A$ in $X$ and a $G$-map $\psi: U \rightarrow Y$ such that $\left.\psi\right|_{A}=f$. If, in addition, one can always take $U=X$, then we say that $Y$ is an equivariant extensor for $X$ (notation: $Y \in G$-AE $(X)$ ). The map $\psi$ is called a $G$-extension of $f$.

A $G$-space $Y$ is called an equivariant absolute neighborhood extensor for the class $G$ - $\mathcal{M}$ (notation: $Y \in G$-ANE) if $Y \in G$-ANE $(X)$ for any $X \in G-\mathcal{M}$.

Similarly, if $Y \in G-\operatorname{AE}(X)$ for any $X \in G-\mathcal{M}$, then $Y$ is called an equivariant absolute extensor for the class $G-\mathcal{M}$ (notation: $Y \in G-\mathrm{AE}$ ).

A $G$-space $Y \in G$ - $\mathcal{M}$ is called a $G$-equivariant absolute neighborhood retract (for the class $G-\mathcal{M}$ ) (notation: $Y \in G$-ANR), provided that for any closed $G$-embedding $Y \hookrightarrow X$ in a $G$-space $X \in G$ - $\mathcal{M}$, there exists a $G$ retraction $r: U \rightarrow Y$, where $U$ is an invariant neighborhood of $Y$ in $X$. If, in addition, one can always take $U=X$, then we say that $Y$ is a $G$-equivariant absolute retract (notation: $Y \in G$-AR). 
We note that, in general, a metrizable $G$-ANE space $Y$ need not be a $G$-ANR, because it may not belong to the class $G$ - $\mathcal{M}$. But if $Y \in G-\mathcal{M}$ and $Y \in G$-ANE, then clearly $Y \in G$-ANR. The converse is also true: for $G$ almost connected this was proved in [10, Remark 5], and the general case is handled in Corollary 3.10 of Section 3.

Let us recall the well known definition of a slice [42, p. 305]:

Definition 2.1. Let $X$ be a $G$-space and $H$ a closed subgroup of $G$. An $H$-invariant subset $S \subset X$ is called an $H$-slice in $X$ if $G(S)$ is open in $X$ and there exists a $G$-equivariant map $f: G(S) \rightarrow G / H$ such that $S=f^{-1}(e H)$. The saturation $G(S)$ is called a tubular set.

If $G(S)=X$, then we say that $S$ is a global $H$-slice of $X$.

One of the most powerful results in the theory of topological transformation groups states (see [42, Proposition 2.3.1]) that if $X$ is a proper $G$-space with $G$ a Lie group, then for any $x \in X$, there exists a $G_{x}$-slice $S$ in $X$ with $x \in S$. This is no longer true when $G$ is not a Lie group (see [7]). Generalizing the case of Lie group actions, in [14] the following approximate version of Palais' Slice Theorem [42, Proposition 2.3.1] for non-Lie group actions was proved, which plays a key role in the proof of Theorem 6.1:

Theorem 2.2 (Approximate Slice Theorem [14]). Let $G$ be a locally compact group, $X$ a proper $G$-space, and $x \in X$. Then for any neighborhood $O$ of $x$ in $X$, there exist a compact large subgroup $K \subset G$ with $G_{x} \subset K$ and a $K$-slice $S$ such that $x \in S \subset O$.

For $G$ compact this theorem was proved in [7]. A version of it, without requiring $K$ to be a large subgroup, was obtained in [2].

We recall that a closed subgroup $H \subset G$ is called large if there exists a closed normal subgroup $N \subset G$ such that $N \subset H$ and $G / N$ is a Lie group.

The quotient space $G / H$ is metrizable for every large subgroup $H \subset G$; this follows from the evident homeomorphism $G / H \cong \frac{G / N}{H / N}$ (see [14, Proposition 4.2]). We shall use this fact in the proof of Theorem 6.1.

Furthermore, it was established in [14] that for a compact subgroup $H \subset$ $G$ the following properties are equivalent: (1) $H$ is a large subgroup; (2) $G / H$ is a metrizable $G$-ANE space; (3) $G / H$ is locally contractible; (4) $G / H$ is $G$ homeomorphic to a smooth $G$-manifold on which $G$ acts by diffeomorphisms (see also [7], [10]).

Observe that every maximal compact subgroup $K$ of an almost connected group $G$ is large. Indeed, Glushkov [29, Theorem 8] proved that each neighborhood of the unity of $G$ contains a compact normal subgroup $N$ such that $G / N$ is a Lie group. By maximality of $K$ one has $N \subset K$, as required.

Lemma 2.3 ([1]). Let $H$ be a compact subgroup of $G, X$ a proper $G$ space, and $f: X \rightarrow G / H$ a $G$-map. Let $S=f^{-1}(e H)$. Then the map 
$\xi: G \times_{H} S \rightarrow X$ defined by $\xi([g, s])=g s$ is a G-homeomorphism, and $\xi f=p$, where the $G$-map $p: G \times_{H} S \rightarrow G / H$ is given by $p([g, s])=g H$ for any $[g, s] \in G \times_{H} S$.

Proposition 2.4 ([10]). Let $G$ be an almost connected group. Then for a closed subgroup $H \subset G$, the following conditions are equivalent:

(1) $H$ is a maximal compact subgroup of $G$;

(2) $G / H$ is a metrizable $G$-AE $(X)$ for every paracompact proper $G$-space $X$ whose orbit space $X / G$ is paracompact.

Lemma 2.5. Let $H$ be a maximal compact subgroup of $G$. Assume that $A$ is a closed invariant subset of a $G$-space $X \in G$-M, and $S$ is a global $H$-slice of $A$. Then there exists a global $H$-slice $\tilde{S}$ in $X$ such that $\tilde{S} \cap A=S$.

Proof. Let $f: A \rightarrow G / H$ be a $G$-map with $f^{-1}(e H)=S$. Since $X \in G$ $\mathcal{M}$, the orbit space $X / G$ is metrizable, and hence paracompact. Consequently, Proposition 2.4(2) yields a $G$-extension $F: X \rightarrow G / H$. It is easy to see that $\tilde{S}=F^{-1}(e H)$ is the desired global $H$-slice.

TheOREM 2.6 ([6], [10]). Let $G$ be a compact group, $H$ a closed normal subgroup of $G$, and $X$ a $G$-AR (respectively, $a G$-ANR). Then $X / H$ is a $G / H$-AR (respectively, $a G / H$-ANR).

We refer to [6, Theorem 8] and [10, Theorem 1] for the details.

3. Equivariant embeddings into proper $G$-AE spaces. Recall that the acting group $G$ is always assumed to be locally compact unless otherwise stated.

In this section we are going to prove the following two equivariant embedding results which solve the linearization problem for proper actions of locally compact groups:

Theorem 3.1. For each $G$-space $X \in G-\mathcal{M}$, there exist a Banach $G$ space $L$ and a $G$-embedding $f: X \hookrightarrow L \backslash\{0\}$ such that $L \backslash\{0\}$ is a proper $G$-space and $L \backslash\{0\} \in H$-AE for any closed subgroup $H$ of $G$.

For purposes of the equivariant theory of retracts and equivariant shape theory it is important to have a $G$-embedding $f$ as in the previous theorem which, in addition, is a closed map. This is achieved in the following

Theorem 3.2. For each $G$-space $X \in G$-M there exist a Banach $G$ space $L$, a normed linear space $N$, and a closed $G$-embedding $f: X \hookrightarrow$ $(L \backslash\{0\}) \times N$ such that $(L \backslash\{0\}) \times N$ is a proper $G$-space and $(L \backslash\{0\}) \times N \in H$ AE for any closed subgroup $H$ of $G$.

REMARK 3.3. One can observe that in contrast to Theorem 3.1, in Theorem 3.2 the "nonproper part" of the ambient $G$-space $E=L \times N$ is not a 
point, but an entire $G$-invariant linear subspace $D=\{0\} \times N$ of $E$. However, $D$ is a $Z$-set in $E$, i.e., for every $\varepsilon>0$ there exists a continuous map $f: E \rightarrow E \backslash D$ such that $\|f(x)-x\|<\varepsilon$ for all $x \in E$. Indeed, since $L$ is infinite-dimensional (as $L \backslash\{0\} \in \mathrm{AE}$ ), the one-point set $\{0\}$ is a $Z$-set in it, which implies immediately that $D$ is a $Z$-set in $E$.

We notice that in [35] M. Kankaanrinta has proved that if $G$ is a Lie group then every smooth proper $G$-manifold admits a smooth $G$-embedding as a closed submanifold of some Hilbert $G$-space.

Below we shall give a sequence of propositions and lemmas culminating in the proofs of Theorems 3.1 and 3.2.

In what follows we denote by $B(x, r)$ the open ball of radius $r$ centered at the point $x$ of a given metric space.

In this section we shall repeatedly use the following condition (some sort of uniform properness) on a normed linear $G$-space $X$ :

$\left(\mathrm{A}_{n}\right)$ for any nonzero points $x, y \in X$, the balls $B(x,\|x\| / n)$ and $B(y,\|x\| / n), n \geq 1$, are relatively thin.

To give a typical example of a normed linear $G$-space that satisfies $\left(\mathrm{A}_{n}\right)$, we first recall that a continuous function $f: X \rightarrow \mathbb{R}$ defined on a $G$-space $X$ is called $G$-uniform if for each $\epsilon>0$ there is a neighborhood $U$ of the unity of $G$ such that $|f(g x)-f(x)|<\epsilon$ for all $x \in X$ and $g \in U$.

For a proper $G$-space $X$ we denote by $\mathcal{P}(X)$ the linear space of all $G$ uniform bounded functions $f: X \rightarrow \mathbb{R}$ whose support supp $f=\{x \in X \mid$ $f(x) \neq 0\}$ is a small subset of $X$. We endow $\mathcal{P}(X)$ with the sup-norm and the following $G$-action:

$$
(g, f) \mapsto g f, \quad(g f)(x)=f\left(g^{-1} x\right), \quad x \in X .
$$

It is easy to see that $\mathcal{P}(X)$ is a normed linear $G$-space. It will play a central role in our further constructions.

The following result is implicit in the proof of [3, Proposition 3.1]:

Proposition 3.4. Let $X$ be a proper $G$-space. Then the $G$-space $\mathcal{P}(X)$ satisfies condition $\left(\mathrm{A}_{4}\right)$.

Proof. Let $f, h \in \mathcal{P}(X) \backslash\{0\}$. To show that the open balls $B(f,\|f\| / 4)$ and $B(h,\|f\| / 4)$ are relatively thin, fix a small set $U$ in $X$ such that $h(x)=0$ whenever $x \in X \backslash U$. Choose $x_{0} \in X$ such that $\left|f\left(x_{0}\right)\right|>3\|f\| / 4$. Since the transporter $\left\langle\left\{x_{0}\right\}, U\right\rangle$ has a compact closure in $G$, it suffices to show that

$$
\langle B(f,\|f\| / 4), B(h,\|f\| / 4)\rangle \subset\left\langle\left\{x_{0}\right\}, U\right\rangle .
$$

To see this, let $g \in\langle B(f,\|f\| / 4), B(h,\|f\| / 4)\rangle$. Then there is $h^{\prime} \in$ $B(h,\|f\| / 4)$ such that $g^{-1} h^{\prime} \in B(f,\|f\| / 4)$. This implies that $\left|h^{\prime}\left(g x_{0}\right)\right| \geq$ 
$\left|f\left(x_{0}\right)\right|-\|f\| / 4$ and $\left|h\left(g x_{0}\right)\right| \geq\left|h^{\prime}\left(g x_{0}\right)\right|-\|f\| / 4$. Thus,

$$
\left|h\left(g x_{0}\right)\right| \geq\left|f\left(x_{0}\right)\right|-\frac{\|f\|}{4}-\frac{\|f\|}{4}>\frac{3\|f\|}{4}-\frac{\|f\|}{4}-\frac{\|f\|}{4}=\frac{\|f\|}{4}>0 .
$$

It follows that $g x_{0} \in U$, i.e., $g \in\left\langle\left\{x_{0}\right\}, U\right\rangle$, as required.

Proposition 3.5. Let $Z$ be a normed linear $G$-space that contains a dense $G$-invariant linear subspace $X$ satisfying condition $\left(\mathrm{A}_{n}\right)$ for some $n \geq 1$. Then $Z \backslash\{0\}$ is a proper $G$-space.

Proof. For any $x \in X$ and $r>0$, we denote by $\widetilde{B}(x, r)$ the open ball in $Z$ centered at $x$ of radius $r$.

Fix $z \in Z \backslash\{0\}$. Since $X$ is dense in $Z$, one can choose $x \in X$ such that $\|x-z\|<\|z\| /(n+1)$. Then $\|z\| /(n+1)<\|x\| / n$, and hence $z \in \widetilde{B}(x,\|x\| / n)$.

We claim that $\widetilde{B}(x,\|x\| / n)$ is a small set in $Z \backslash\{0\}$. Indeed, for any $z^{\prime} \in$ $Z \backslash\{0\}$, choose $y \in X$ such that $\left\|y-z^{\prime}\right\|<\|x\| / n$. Then $z^{\prime} \in \widetilde{B}(y,\|x\| / n)$.

Let us check that $\widetilde{B}(x,\|x\| / n)$ and $\widetilde{B}(y,\|x\| / n)$ are relatively thin. Assume that $g$ belongs to the transporter $\langle\widetilde{B}(x,\|x\| / n), \widetilde{B}(y,\|x\| / n)\rangle$. Then $g \widetilde{B}(x,\|x\| / n) \cap \widetilde{B}(y,\|x\| / n) \neq \emptyset$. Since $X \backslash\{0\}$ is dense in $Z \backslash\{0\}$ we infer that

$$
(X \backslash\{0\}) \cap g \widetilde{B}(x,\|x\| / n) \cap \widetilde{B}(y,\|x\| / n) \neq \emptyset
$$

But

$$
\begin{gathered}
(X \backslash\{0\}) \cap g \widetilde{B}(x,\|x\| / n)=g B(x,\|x\| / n), \\
(X \backslash\{0\}) \cap \widetilde{B}(y,\|x\| / n)=B(y,\|x\| / n) .
\end{gathered}
$$

Hence, (3.1) reads

$$
g B(x,\|x\| / n) \cap B(y,\|x\| / n) \neq \emptyset,
$$

showing that $g \in\langle B(x,\|x\| / n), B(y,\|x\| / n)\rangle$. Thus, we have proved that

$$
\langle\widetilde{B}(x,\|x\| / n), \widetilde{B}(y,\|x\| / n)\rangle \subset\langle B(x,\|x\| / n), B(y,\|x\| / n)\rangle .
$$

It remains to observe that $\langle B(x,\|x\| / n), B(y,\|x\| / n)\rangle$ has a compact closure in $G$, by hypothesis.

Below we shall consider the following situation. Let $G$ be any group and $\left\{E_{i} \mid i \in \mathcal{I}\right\}$ a family of normed linear $G$-spaces. Denote by $E$ the $\sigma$-product of $\left\{E_{i} \mid i \in \mathcal{I}\right\}$, i.e., $E=\left\{v=\left(v_{i}\right) \in \prod_{i \in \mathcal{I}} E_{i} \mid v_{i} \neq 0\right.$ for finitely many $i \in \mathcal{I}\}$. We consider the following norm on $E$ :

$$
\|v\|=\sum_{i \in \mathcal{I}}\left\|v_{i}\right\|, \quad \text { where } \quad v=\left(v_{i}\right) \in E .
$$

Proposition 3.6. Let $\left\{E_{i} \mid i \in \mathcal{I}\right\}$ be a family of normed linear $G$ spaces such that each $E_{i}$ satisfies condition $\left(\mathrm{A}_{4}\right)$. Then the $\sigma$-product $E$ of 
$\left\{E_{i} \mid i \in \mathcal{I}\right\}$, endowed with the diagonal action of $G$, becomes a normed linear $G$-space that satisfies condition $\left(\mathrm{A}_{8}\right)$.

Proof. To check the continuity of the action of $G$ on $E$, let $\varepsilon>0, u \in E$ and $h \in G$. Assume that $i_{1}, \ldots, i_{m}$ are all the indices such that $u_{i_{k}} \neq 0$. By continuity of the action of $G$ on $E_{i_{k}}, k=1, \ldots, m$, one can find a neighborhood $O$ of $h$ in $G$ such that

$$
\left\|g u_{i_{k}}-h u_{i_{k}}\right\|<\varepsilon / 2 m \quad \text { for all } g \in O \text { and } k=1, \ldots, m .
$$

This yields

$$
\|g u-h u\|=\sum_{i \in \mathcal{I}}\left\|g u_{i}-h u_{i}\right\|=\sum_{k=1}^{m}\left\|g u_{i_{k}}-h u_{i_{k}}\right\|<\sum_{k=1}^{m} \varepsilon / 2 m=\varepsilon / 2
$$

for all $g \in O$.

On the other hand, the action of $G$ on $E$ is isometric, i.e., $\|g v\|=\|v\|$ for all $v \in E$ and $g \in G$.

Consequently, for every $g \in O$ and $v \in B(u, \varepsilon / 2)$ one has

$$
\begin{aligned}
\|g v-h u\| & \leq\|g v-g u\|+\|g u-h u\| \\
& =\|v-u\|+\|g u-h u\|<\varepsilon / 2+\varepsilon / 2=\varepsilon,
\end{aligned}
$$

as required.

Now let us check $\left(\mathrm{A}_{8}\right)$ in $E$. Fix $v=\left(v_{i}\right) \in E$. Assume that $i_{1}, \ldots, i_{m}$ are all the indices such that $v_{i_{k}} \neq 0$. Let us check that for every $u \in E$,

$$
\langle B(v,\|v\| / 8), B(u,\|v\| / 8)\rangle \subset \bigcup_{k=1}^{m}\left\langle B\left(v_{i_{k}},\left\|v_{i_{k}}\right\| / 4\right), B\left(u_{i_{k}},\left\|v_{i_{k}}\right\| / 4\right)\right\rangle .
$$

Indeed, let $g \in\langle B(v,\|v\| / 8), B(u,\|v\| / 8)\rangle$. Then

$$
g B(v,\|v\| / 8) \cap B(u,\|v\| / 8) \neq \emptyset .
$$

Consequently, there exists $z \in E$ with $\|z-v\|<\|v\| / 8$ such that $\|g z-u\|<$ $\|v\| / 8$. These inequalities imply that

$$
\begin{gathered}
\sum_{k=1}^{m}\left\|z_{i_{k}}-v_{i_{k}}\right\|<\sum_{k=1}^{m}\left\|v_{i_{k}}\right\| / 8, \\
\sum_{k=1}^{m}\left\|g z_{i_{k}}-u_{i_{k}}\right\|<\sum_{k=1}^{m}\left\|v_{i_{k}}\right\| / 8 .
\end{gathered}
$$

Summing up these two inequalities we obtain

$$
\sum_{k=1}^{m}\left(\left\|z_{i_{k}}-v_{i_{k}}\right\|+\left\|g z_{i_{k}}-u_{i_{k}}\right\|\right)<\sum_{k=1}^{m}\left\|v_{i_{k}}\right\| / 4 .
$$

Hence, there exists an index $1 \leq j \leq m$ (depending on $g$ ) such that

$$
\left\|z_{i_{j}}-v_{i_{j}}\right\|+\left\|g z_{i_{j}}-u_{i_{j}}\right\|<\left\|v_{i_{j}}\right\| / 4 \text {. }
$$


In particular,

$$
\left\|z_{i_{j}}-v_{i_{j}}\right\|<\left\|v_{i_{j}}\right\| / 4 \text { and }\left\|g z_{i_{j}}-u_{i_{j}}\right\|<\left\|v_{i_{j}}\right\| / 4
$$

which means that $g z_{i_{j}} \in g B\left(v_{i_{j}},\left\|v_{i_{j}}\right\| / 4\right) \cap B\left(u_{i_{j}},\left\|v_{i_{j}}\right\| / 4\right)$. Thus,

$$
g B\left(v_{i_{j}},\left\|v_{i_{j}}\right\| / 4\right) \cap B\left(u_{i_{j}},\left\|v_{i_{j}}\right\| / 4\right) \neq \emptyset
$$

yielding

$$
g \in\left\langle B\left(v_{i_{j}},\left\|v_{i_{j}}\right\| / 4\right), B\left(u_{i_{j}},\left\|v_{i_{j}}\right\| / 4\right)\right\rangle,
$$

as required. Thus (3.2) holds.

Since every $E_{i}$ satisfies condition $\left(\mathrm{A}_{4}\right)$, each transporter $\left\langle B\left(v_{i_{k}},\left\|v_{i_{k}}\right\| / 4\right)\right.$, $\left.B\left(u_{i_{k}},\left\|v_{i_{k}}\right\| / 4\right)\right\rangle, k=1, \ldots, m$, has a compact closure in $G$. By (3.2), so does $\langle B(v,\|v\| / 8), B(u,\|v\| / 8)\rangle$, which means that $B(v,\|v\| / 8)$ and $B(u,\|v\| / 8)$ are relatively thin.

Corollary 3.7. Let $\left\{E_{i} \mid i \in \mathcal{I}\right\}$ and $E$ be as in Proposition 3.6, and let $\widetilde{E}$ be the completion of $E$. Then $\widetilde{E} \backslash\{0\}$ is a proper $G$-space.

Proof. Since the action of $G$ on $E$ is isometric, it can be uniquely extended to an isometric action of $G$ on $\widetilde{E}$. Let us check the continuity of this action.

Indeed, let $\left(g_{0}, x_{0}\right) \in G \times \widetilde{E}$ and $\varepsilon>0$. Choose $v_{0} \in E$ with $\left\|v_{0}-x_{0}\right\|<$ $\varepsilon / 4$. By continuity of the action on $E$, there exists a neighborhood $O$ of $g_{0}$ in $G$ such that $\left\|g v_{0}-g_{0} v_{0}\right\|<\varepsilon / 4$. On the other hand,

$$
\begin{gathered}
\left\|g x-g x_{0}\right\|=\left\|x-x_{0}\right\|, \quad\left\|g x_{0}-g v_{0}\right\|=\left\|x_{0}-v_{0}\right\|, \\
\left\|g_{0} v_{0}-g_{0} x_{0}\right\|=\left\|v_{0}-x_{0}\right\| .
\end{gathered}
$$

Hence

$$
\begin{aligned}
\left\|g x-g_{0} x_{0}\right\| \leq & \left\|g x-g x_{0}\right\|+\left\|g x_{0}-g v_{0}\right\|+\left\|g v_{0}-g_{0} v_{0}\right\| \\
& +\left\|g_{0} v_{0}-g_{0} x_{0}\right\| \\
= & \left\|x-x_{0}\right\|+\left\|x_{0}-v_{0}\right\|+\left\|g v_{0}-g_{0} v_{0}\right\|+\left\|v_{0}-x_{0}\right\| \\
< & \varepsilon / 4+\varepsilon / 4+\varepsilon / 4+\varepsilon / 4=\varepsilon
\end{aligned}
$$

whenever $g \in O$ and $\left\|x-x_{0}\right\|<\varepsilon / 4$. This proves the continuity of the action of $G$ on $\widetilde{E}$, and hence $\widetilde{E}$ is a normed linear $G$-space. Now it follows immediately from Propositions 3.5 and 3.6 that $\widetilde{E} \backslash\{0\}$ is a proper $G$-space.

Proposition 3.8. Let $E$ be a Banach $G$-space such that for each compact subgroup $H \subset G$, the $H$-fixed point set $E^{H}$ is infinite-dimensional. Then $E \backslash\{0\}$ is a $G$-AE.

Proof. We aim at applying the following result of Abels [2, Theorem 4.4]: a $G$-space $T$ is a $G$-AE if $T$ is a $K$-AE for each compact subgroup $K \subset G$. 
In our case $T=E \backslash\{0\}$. In order to show that for each compact subgroup $K \subset G, E \backslash\{0\}$ is a $K$-AE, we will apply the following generalization of the James-Segal Theorem [34, Proposition 4.1]:

TheOrem (see [16]). Let $K$ be a compact group and $T$ a $K$-ANR. Then $T$ is a K-AR if and only if for every closed subgroup $H \subset K$ the set of $H$-fixed points $T^{H}=\{t \in T \mid h t=t, \forall h \in H\}$ is contractible.

We continue with the proof of Proposition 3.8. By completeness of $E$, the equivariant Dugundji extension theorem, as proved in [4], shows that $E$ is a $K$-AR. Hence, $E \backslash\{0\}$ is a $K$-ANR.

Let $H \subset K$ be any closed subgroup. Since $E^{H}$ is an infinite-dimensional normed linear space, according to a result of Klee [36], $E^{H} \backslash\{0\}$ is homeomorphic to $E^{H}$, and hence is contractible.

Since $(E \backslash\{0\})^{H}=E^{H} \backslash\{0\}$ we infer that $(E \backslash\{0\})^{H}$ is contractible, as required. Now the above theorem of [16] implies that $E \backslash\{0\}$ is a $K$-AR, which completes the proof.

Proof of Theorem 3.1. First we assume that $G$ is not compact. It is established in the proof of [3, Theorem 3.9] that then there exist normed linear $G$-spaces $\left\{E_{i} \mid i \in \mathcal{I}\right\}$ satisfying condition $\left(\mathrm{A}_{4}\right)$ and a $G$-embedding $\varphi: X \hookrightarrow E \backslash\{0\}$, where $E$ is the $\sigma$-product of $\left\{E_{i} \mid i \in \mathcal{I}\right\}$. Recall that the $G$-spaces $E_{i}$ in question are all normed linear $G$-spaces of the form $\mathcal{P}\left(Y_{i}\right)$, considered in Proposition 3.4.

By Corollary 3.7, the completion $L$ of $E$ is a Banach $G$-space and $L \backslash\{0\}$ is a proper $G$-space. Then the composition $f=j \varphi$,

$$
X \stackrel{\varphi}{\hookrightarrow} E \backslash\{0\} \stackrel{j}{\hookrightarrow} L \backslash\{0\},
$$

is the desired $G$-embedding, where $j: E \backslash\{0\} \hookrightarrow L \backslash\{0\}$ is the natural inclusion.

Now we pass to the property $L \backslash\{0\} \in H$-AE for any closed subgroup $H$ of $G$. It is established in the proof of [3, Theorem 3.9] that for each compact subgroup $K \subset G$, the $K$-fixed point set $E^{K}$ is an infinite-dimensional closed subspace of $E$. This implies that $L^{K}$ is also an infinite-dimensional closed subspace of $L$. Now it follows from Proposition 3.8 that $L \backslash\{0\} \in H$-AE. This completes the proof in the case of $G$ noncompact.

Next we assume that $G$ is compact. It is known (see [5, Theorem 1]) that $X$ can be $G$-embedded in $L \backslash\{0\}$ for some Banach $G$-space $L$ (possibly finite-dimensional). Take an arbitrary infinite-dimensional Banach space $Z$ (say, $Z=\ell_{2}$, the Hilbert space) with the trivial action of $G$. Then the map $l \mapsto(l, 0), l \in L$, is a $G$-embedding of $L$ into $E=L \times Z$, which is an infinite-dimensional Banach $G$-space. As a result, $X$ is $G$-embedded in $E \backslash\{0\}$. By compactness of $G, E \backslash\{0\}$ is a proper $G$-space. On the other hand, $E^{K}=L^{K} \times Z$ for any compact subgroup $K \subset H$, implying 
that $E^{K}$ is an infinite-dimensional Banach space. Now Proposition 3.8 yields $E \backslash\{0\} \in H$-AE.

For the proof of Theorem 3.2 we shall need the following lemma proved in [3]:

Lemma 3.9. Let $f: X \rightarrow M$ be a $G$-map between two proper $G$-spaces and let $p: X \rightarrow X / G$ be the orbit map. Then the image of the diagonal map $\varphi: X \rightarrow M \times(X / G), \varphi(x)=(f(x), p(x))$, is a closed invariant subset of $M \times(X / G)$ endowed with the diagonal $G$-action, where $X / G$ is equipped with the trivial $G$-action.

Proof of Theorem 3.2. Let $j: X \hookrightarrow L \backslash\{0\}$ be the $G$-embedding from Theorem 3.1 and let $p: X \rightarrow X / G$ be the orbit map. Then the diagonal product of $j$ and $p$ is a topological embedding

$$
\varphi: X \hookrightarrow(L \backslash\{0\}) \times(X / G)
$$

(see e.g. [26, Theorem 2.3.20]). Clearly $\varphi$ is equivariant.

Next, it follows from Lemma 3.9 that $\varphi$ is a closed embedding. Thus, one can think of $X$ as a closed invariant subset of $(L \backslash\{0\}) \times(X / G)$. But $X / G$ is metrizable (see Section 2), and hence, according to the Arens-Eells embedding theorem (see e.g. [19, Ch. II, Corollary 1.1]), one can embed $X / G$ into a normed linear space $N$ as a closed subset.

This generates a closed equivariant embedding of $(L \backslash\{0\}) \times(X / G)$ into $(L \backslash\{0\}) \times N$. As a result we get an equivariant closed embedding of $X$ into $(L \backslash\{0\}) \times N$. Since the product of a proper $G$-space with any $G$-space is again a proper $G$-space we see that $(L \backslash\{0\}) \times N$ is a proper $G$-space.

By Theorem 3.1, $L \backslash\{0\} \in H$-AE for any closed subgroup $H$ of $G$. By the Dugundji extension theorem [23], $N \in \mathrm{AE}$, and hence $N$ endowed with the trivial $H$-action is an $H$-AE (see e.g. [3, Lemma 3.12]). Since the product of two $H$-AE spaces is again an $H$-AE space (this is quite easy to check), we conclude that $(L \backslash\{0\}) \times N \in H$-AE.

The following result, in a particular case when $G$ is almost connected, was obtained in [10, Remark 5]; the case when $G$ is a Lie group is treated also in [3] and [27]:

Corollary 3.10. Let $X \in G$-M. Then $X$ is a $G$-ANE (respectively, $a G$-AE) if and only if $X$ is a $G$-ANR (respectively, $a G$-AR).

Proof. We consider the " $G$-AR" case only; the " $G$-ANR" case is quite similar.

As we noticed in Section 2, if $X \in G$-M and $X$ is a $G$-AE, then clearly $X$ is a $G$-AR. Now suppose that $X$ is a $G$-AR. Then by Theorem 3.2, one can think of $X$ as a closed invariant subset of a $G$-space $L \in G$ - $\mathcal{M}$ which is 
a $G$-AE. Since $X$ is a $G$-AR, it is an equivariant retract of $L$, which implies immediately that $X$ is a $G$-AE.

Corollary 3.11. Let $X$ be a $G$-ANR (respectively, a $G$-AR) and $H \subset G$ a closed subgroup. Then $X$ is an $H$-ANR (respectively, an $H$-AR).

Proof. We consider the "G-AR" case only; the "G-ANR" case is quite similar.

By Theorem 3.2, one can think of $X$ as a closed invariant subset of a proper $G$-space $(L \backslash\{0\}) \times N$ with $L$ and $N$ as in Theorem 3.2. Since $X$ is a $G$-AR, it is a $G$-equivariant retract (and, in particular, an $H$-equivariant retract) of $(L \backslash\{0\}) \times N$. Since $(L \backslash\{0\}) \times N \in H$-AR we infer that $X \in H$ AR.

4. Orbit spaces of $G$-AR spaces. In this section we shall prove the following special case of Theorem 7.1 which is an essential step in the proof of the latter:

TheOREM 4.1. Let $G$ be a locally compact group and $X \in G$-AR. Assume that $H$ is an almost connected normal subgroup of $G$. Then $X / H$ is a $G / H$ AE.

We note that almost connectedness of $H$ is essential in this theorem. Indeed, let $G=\mathbb{R}, X=\mathbb{R}$, and $H=\mathbb{Z}$. Then the translation action is a proper action of $G$ on $X$, and by [2, Theorem 4.4], $X$ is a $G$-AE. However, $X / H$, being a circle, is not an AE.

Theorem 4.1 just extends Theorem 2.6 to the case of arbitrary locally compact proper group actions. For its proof we need several auxiliary results.

Proposition 4.2. Let $G$ be an almost connected group, $K$ a maximal compact subgroup of $G$, and $S$ a $K$-space. Then the twisted product $G \times_{K} S$ is a $G-\mathrm{AE}$ if and only if $S$ is a $K-\mathrm{AE}$.

Proof. To proove the "if" part, let $Z \in G-\mathcal{M}, C$ be a closed $G$-invariant subset of $Z$, and $f: C \rightarrow G \times{ }_{K} S$ a $G$-map. It is well known and easy to check that $f^{-1}(S)$ is a global $K$-slice of $C$ (see e.g. [41, Corollary 1.7.8]). By Lemma 2.5 , there is a global $K$-slice $Y$ in $Z$ such that $Y \cap C=f^{-1}(S)$. Consequently, $f^{-1}(S)$ is a closed $K$-invariant subset of the metrizable $K$-space $Y$. Since $S$ is a $K$-AE, there exists a $K$-extension $f_{1}: Y \rightarrow S$ of the $K$-map $\left.f\right|_{f^{-1}(S)}$ : $f^{-1}(S) \rightarrow S$. Then $f_{1}$ induces a $G$-map $F: Z=G(Y) \rightarrow G \times_{H} S$ by $F(g y)=g f_{1}(y)$, where $g \in G$ and $y \in Y$ (see [22, Ch. I, Proposition 4.3]). If $c \in C$, then $c=g b$ for some $g \in G$ and $b \in f^{-1}(S)$, and hence

$$
F(c)=F(g b)=g f_{1}(b)=g f(b)=f(g b)=f(c) .
$$

Thus, $F$ extends $f$, and the proof of the "if" part is complete. 
The "only if" part follows from two facts. The first, [15, Proposition 3.5], states that $G \times{ }_{K} S$ is a $G$-AE if and only if it is a $K$-AE (remember that a maximal compact subgroup is large, as observed in Section 2). The second fact is that the global $K$-slice $S$ is a $K$-equivariant retract of $G \times{ }_{K} S$; this follows from a result of Abels [1, Theorem 2.1] according to which $G \times{ }_{K} S$ is $K$-homeomorphic to a product $T \times S$ endowed with the diagonal action of $K$, where $T$ is a finite-dimensional linear $K$-space. In this case the map $(t, s) \mapsto(0, s)$ is a $K$-equivariant retraction of $T \times S$ onto $\{0\} \times S$, which, in turn, is $K$-homeomorphic to $S$.

Lemma 4.3 ([15]). Let $G$ be a locally compact group, $H$ a closed normal subgroup of $G$, and $K$ a compact subgroup of $G$. Then the map

defined by

$$
f: K H / H \rightarrow K /(K \cap H)
$$

$$
f(k H)=k(K \cap H), \quad \text { where } k \in K \text { and } k H \in K H / H,
$$

is a topological isomorphism.

Proposition 4.4. Let $G$ be a locally compact group, $H$ a closed normal subgroup of $G, K$ a compact subgroup of $G$, and $S$ a $K$-space. Then one has the following $G / H$-homeomorphism:

$$
\left(G \times_{K} S\right) / H \cong \frac{G}{H} \times_{K H / H} \frac{S}{K \cap H} .
$$

Proof. Using the topological isomorphism $K H / H \cong K /(K \cap H)$ from Lemma 4.3, we shall consider the $(K \cap H)$-orbit space $S /(K \cap H)$ as a $K H / H$-space. Specifically, the group $K H / H$ acts on $S /(K \cap H)$ according to the rule

$$
(k H) *(K \cap H)(s)=(K \cap H)(k s)
$$

for $k \in K$ and $(K \cap H)(s) \in S /(K \cap H)$.

The required canonical $G / H$-homeomorphism

$$
f:\left(G \times_{K} S\right) / H \rightarrow \frac{G}{H} \times_{K H / H} \frac{S}{K \cap H}
$$

is given by $f(H([g, s]))=[g H, \tilde{s}]$, where $H([g, s])$ denotes the $H$-orbit of $[g, s]$ in the $G$-space $G \times_{K} S, \tilde{s}$ stands for the $(K \cap H)$-orbit of $s$ in the $K$ space $S$, and $[g H, \tilde{s}]$ denotes the $K H / H$-orbit of $(g H, \tilde{s})$ in the $K H / H$-space $\frac{G}{H} \times \frac{S}{K \cap H}$. It is easy to verify that $f$ is as desired.

Proof of Theorem 4.1. Case 1. Assume that $G$ is almost connected. Since $X \in G$ - $\mathcal{M}$, by [1, Main Theorem], $X$ admits a global $K$-slice $S$, where $K$ is a maximal compact subgroup of $G$. Hence, by Lemma 2.3, $X=G \times_{K} S$. By Proposition 4.4, one has the $G / H$-homeomorphism

$$
X / H \cong G / H \times_{K^{\prime}} S / H^{\prime},
$$

where $H^{\prime}=K \cap H$ and $K^{\prime}=K H / H$. 
Since by Corollary 3.10, $X \in G$-AE, it follows from Proposition 4.2 that $S$ is a $K$-AE. Hence, Theorem 2.6 shows that $S / H^{\prime}$ is a $K /(K \cap H)$-AE. Since the group $K^{\prime}$ acts on $S / H^{\prime}$ via the topological isomorphism $K^{\prime} \cong K /(K \cap H)$, we infer that $S / H^{\prime}$ is a $K^{\prime}$-AE.

Next, by Theorem 8.6 (see Appendix) the group $K^{\prime}$, being the image of $K$ under the natural homomorphism $\pi: G \rightarrow G / H$, is a maximal compact subgroup of $G / H$. Hence, Proposition 4.2 shows that $G / H \times_{K^{\prime}} S / H^{\prime}$ is a $G / H$-AE. It remains to invoke the formula (4.2).

Case 2. Assume that $G$ is arbitrary locally compact. According to [2, Theorem 4.4], it suffices to show that $X / H$ is a $K$-AE for any compact subgroup $K$ of $G / H$. Consider the natural homomorphism $\pi: G \rightarrow G / H$. By Proposition 8.3 (see Appendix), the group $M=\pi^{-1}(K)$ is almost connected.

Since $X \in G$-AR it follows that $X \in M$-AR (see Corollary 3.11). Hence $X / H$ is an $M / H$-AE by Case 1 . Since $M / H=K$, we infer that $X \in K$-AE, as required.

Recall that the "G-ANR" case of Theorem 4.1 was established earlier in [15, Theorem 1.1]; here is its exact formulation:

THEOREM 4.5. Let $G$ be a locally compact group, $X \in G$-ANR, and $H$ a closed normal subgroup of $G$. Then $X / H$ is a $G / H$-ANE.

REMARK 4.6. Theorem 4.1 is not valid in the category of compact (nonmetrizable) $G$-spaces even if $G$ is a finite group. Here is a simple counterexample.

Consider the antipodal action of the cyclic group $G=\mathbb{Z}_{2}$ on the Tikhonov cube $I^{\tau}$ of an uncountable weight $\tau$, where $I=[-1,1]$. Since $I$ is a $G$-AE for normal $G$-spaces, we infer that $I^{\tau}$ is a $G$-AR for compact $G$-spaces. However, $I^{\tau} / G$ is not an AR for compact spaces. This follows from the following result of Shchepin [44]: if $X$ is a nonmetrizable AR in the category of compact Hausdorff spaces, then it is homeomorphic to a Tikhonov cube $I^{\tau}$ if and only if $X$ is homogeneous with respect to character (i.e., all the points have the same character in $X)$. Now suppose that $I^{\tau} / G$ is an AR for compact spaces. Since obviously $I^{\tau} / G$ is homogeneous with respect to character, it should be homeomorphic to $I^{\tau}$. But this is impossible, because, unlike the cube $I^{\tau}$, the orbit space $I^{\tau} / G$ contains a point whose complement is not contractible. In fact, let $\theta$ be the point of $I^{\tau}$ with all coordinates 0 . Then the orbit projection

$$
p: I^{\tau} \backslash\{\theta\} \rightarrow\left(I^{\tau} \backslash\{\theta\}\right) / G
$$

is a two-sheet covering. Since $I^{\tau} \backslash\{\theta\}$ is contractible, the fundamental group of $\left(I^{\tau} \backslash\{\theta\}\right) / G$ is $G=\mathbb{Z}_{2}$. Thus $\left(I^{\tau} / G\right) \backslash\{\theta\}=\left(I^{\tau} \backslash\{\theta\}\right) / G$ is not contractible, which implies that $I^{\tau} / G$ is not homeomorphic to a Tikhonov cube. 
5. Equivariant lifting of closed embeddings. The following result for $G$ compact was established in [8]. As in that case, Theorem 4.1 is equivalent to Theorem 5.1 below. In this section we proof the implication Theorem $4.1 \Rightarrow$ Theorem 5.1, while the converse is proved in Section 7 .

THEOREM 5.1. Let $H$ be an almost connected normal subgroup of $G$. Suppose that $A \in G-\mathcal{M}$ and $f: A / H \hookrightarrow B$ is a $G / H$-equivariant closed embedding into a $G / H$-space $B \in G / H-\mathcal{M}$. Then there exist a $G$-space $Z \in$ $G-\mathcal{M}$ and a $G$-equivariant closed embedding $\phi: A \hookrightarrow Z$ such that $Z / H=B$ and $q \circ \phi=f \circ p$, where $p: A \rightarrow A / H$ and $q: Z \rightarrow Z / H$ are the $H$-orbit maps.

Proof. According to Theorem 3.2, it can be assumed that $A$ is a closed invariant subset of a $G$-AE space $L \in G-\mathcal{M}$. Then $A / H$ is a closed invariant subset of the $G / H$-space $L / H$.

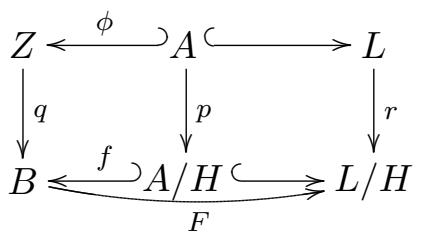

Now, by Theorem 4.1, $L / H \in G / H$-AE. Therefore, there exists a $G / H$ equivariant extension $F: B \rightarrow L / H$ of the $G / H$-map $f^{-1}: f(A / H) \rightarrow$ $A / H \hookrightarrow L / H$. Let $r: L \rightarrow L / H$ be the $H$-orbit projection. Denote by $Z$ the fiber product of the maps $F$ and $r$, i.e.,

$$
Z=\{(b, x) \in B \times L \mid F(b)=r(x)\} .
$$

We consider the diagonal action of $G$ on $Z$, i.e., $g(b, x)=(g b, g x)$ for $g \in G$ and $(b, x) \in Z$. Let $h: Z / H \rightarrow B$ be defined by $h(q(b, x))=b$, where $q: Z \rightarrow Z / H$ is the $H$-orbit projection and $(b, x) \in Z$. It is clear that $h$ is a well-defined $G / H$-equivariant map. It can easily be shown (and is well known, see [31, Ch. 4, Proposition 4.1]) that $h$ is a homeomorphism.

On the other hand, $B \times L$ is a proper $G$-space because $L$ is. Moreover, since $B$ and $L$ admit $G$-invariant metrics, so does $B \times L$. Thus $B \times L \in G$-M , which implies that $Z \in G-\mathcal{M}$.

It remains to define the $G$-equivariant embedding $\phi: A \hookrightarrow Z$ by the formula $\phi(a)=(f(p(a)), a)$ for $a \in A$.

TheOrem 5.2. Let $H$ be any closed normal subgroup of $G$. Suppose that $A \in G$-M and $f: A / H \hookrightarrow B$ is a $G / H$-equivariant closed embedding into a $G / H$-space $B \in G / H-\mathcal{M}$. Then there exist a $G$-space $Z \in G-\mathcal{M}$ and $a G$ equivariant closed embedding $\phi: A \hookrightarrow Z$ such that $Z / H$ is a $G / H$-invariant neighborhood of $A / H$ in $B$ and $q \circ \phi=f \circ p$, where $p: A \rightarrow A / H$ and $q: Z \rightarrow Z / H$ are the $H$-orbit maps. 
The proof of this theorem is quite similar to the one of Theorem 5.1; one has only to refer to Theorem 4.5 instead of Theorem 4.1.

The following special case of Theorems 5.1 and 5.2 when $H=G$, for closed embeddings in a paracompact space, is worth singling out:

Corollary 5.3. Let $G$ be a locally compact (respectively, almost connected) group. Suppose that $A \in G-\mathcal{M}$ and $f: A / G \hookrightarrow B$ is a closed embedding into a paracompact space $B$. Then there exist a paracompact proper $G$-space $Z$ and $a G$-equivariant closed embedding $\phi: A \hookrightarrow Z$ such that $Z / G$ is a closed neighborhood of $A / G$ in $B$ (respectively, $Z / G=B$ ) and $q \circ \phi=f \circ p$, where $p: A \rightarrow A / G$ and $q: Z \rightarrow Z / G$ are the orbit maps.

Proof. Since $A / G$ is metrizable, there exists an embedding $i$ of $A / G$ in the Banach space $Y$ of all continuous bounded real-valued functions over $A / G$ (see e.g. [19, Ch. II, Proposition 1.1]).

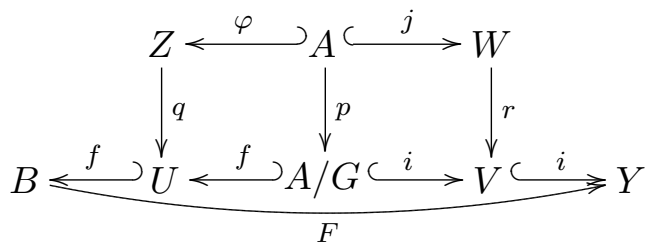

By the Dugundji extension theorem [23], $Y$ is an AR. Consequently, by a result of E. Michael [39], $Y$ is an AE for all paracompact spaces. This implies that $f^{-1}: f(A / G) \rightarrow A / G \hookrightarrow Y$ extends to a continuous map $F: B \rightarrow Y$. Next, by Theorem 5.2 (resp., Theorem 5.1), there exists a neighborhood $V$ of $A / G$ in $Y$ (resp., $V=Y$ ) such that the closed embedding $i: A / G \hookrightarrow V$ admits a $G$-equivariant lifting $j: A \hookrightarrow W$ to a $G$-space $W \in G$ - $\mathcal{M}$.

Choose a closed neighborhood $U$ of $A / G$ in $B$ such that $F(U) \subset V$ (resp., $U=B)$. As in the proof of Theorem 5.1, denote by $Z$ the fiber product of $F: U \rightarrow V$ and $r: W \rightarrow V$, i.e.,

$$
Z=\{(u, w) \in U \times W \mid F(u)=r(w)\} .
$$

Then proceeding as in the proof of Theorem 5.1, we can show that $Z$ is the required proper $G$-space. It is paracompact because $Z / G=U$ is (see [2, Theorem 1.12]).

Remark 5.4. If $A$ is not metrizable, Corollary 5.3 may fail to be true even if $G$ is finite and $A$ and $B$ are compact. Indeed, consider the antipodal action of $G=\mathbb{Z}_{2}$ on the Tikhonov cube $I^{\tau}$ of an uncountable weight $\tau$, where $I=[-1,1]$. Then no embedding $i: I^{\tau} / G \hookrightarrow I^{\tau}$ can be lifted to a $G$-embedding $j: I^{\tau} \hookrightarrow Z$ where $Z$ is a compact $G$-space with $Z / G=I^{\tau}$. Indeed, assume otherwise. Then, since $I^{\tau}$ is a $G$-AE for compact $G$-spaces, there exists a $G$-retraction $r: Z \rightarrow I^{\tau}$. Clearly, $r$ induces a retraction $q$ : 
$I^{\tau}=Z / G \rightarrow I^{\tau} / G$, which implies that $I^{\tau} / G$ is an absolute retract for compact spaces, contradicting Remark 4.6.

\section{Metrizability of proper $G$-spaces}

THeOREM 6.1. Let $G$ be a locally compact group and $X$ a proper $G$-space such that all the orbits in $X$, as well as the orbit space $X / G$, are metrizable. Then $X$ is metrizable. Moreover, there exists a compatible $G$-invariant metric on $X$.

Notice that for $G$ compact metrizable this was proved by B. A. Pasynkov [43, Theorem 2], and for $G$ any compact group by V. V. Filippov [28, Theorem (b)]:

Theorem 6.2 (V. V. Filippov). Let $G$ be a compact group and $X$ a $G$-space such that all the orbits in $X$, as well as the orbit space $X / G$, are metrizable. Then $X$ is metrizable.

Proof. A simple combination of the Nagata-Smirnov metrization theorem [26, Theorem 4.4.7] and V. V. Filippov's result [28, Theorem (b)].

Our proof of Theorem 6.1 is based on the following three lemmas:

Lemma 6.3. Let $G$ be a topological group, and $H$ and $N$ subgroups of $G$ such that $N \subset H$. If $H / N$ and $G / H$ have countable open bases at each point, so does $G / N$.

Proof. This is proved in [32, p. 47] for $N$ being the trivial subgroup of $G$. That proof can easily be adapted to the general case. For the convenience of the reader we provide the details below.

By homogeneity it suffices to show that $G / N$ has a countable open base at $e N \in G / N$.

For any $A \subset G$ we denote by $\widetilde{A}$ the image of $A$ under the natural projection $G \rightarrow G / N$. Since the projection is a $G$-map, one has $\widetilde{g A}=g \widetilde{A}$ for every $g \in G$. This yields $\widetilde{B A}=B \widetilde{A}$ for every $B \subset G$.

Let $\left\{W_{n}\right\}_{n=1}^{\infty}$ be a sequence of symmetric neighborhoods of $e$ in $G$ such that $W_{n+1}^{2} \subset W_{n}$ for each $n$ and such that $\left\{\widetilde{W}_{n} \cap \widetilde{H}\right\}_{n=1}^{\infty}$ is an open base at $e N$ for the space $\widetilde{H}$.

Let $\varphi$ denote the natural map $G \rightarrow G / H$. Suppose that $\left\{\varphi\left(U_{n}\right)\right\}_{n=1}^{\infty}$ is an open base at $e H$ in $G / H$, where the $U_{n}$ are neighborhoods of $e$ in $G$. For $n=1,2, \ldots$, we put

$$
P_{n}=\left(\widetilde{G} \backslash \overline{W_{n+1}\left(\widetilde{H} \backslash \widetilde{W}_{n}\right)}\right) \cap\left(U_{n} \widetilde{H}\right) .
$$

Let us prove that $P_{n}$ is a neighborhood of $e N$ in $\widetilde{G}$. In fact, it suffices to show that $e N \notin \overline{W_{n+1}\left(\widetilde{H} \backslash \widetilde{W}_{n}\right)}$. 
To this end, let us prove that the neighborhood $\widetilde{W}_{n+1}$ of $e N$ does not meet $W_{n+1}\left(\widetilde{H} \backslash \widetilde{W}_{n}\right)$. Assume otherwise. Then $x N=w h N$ with $x \in W_{n+1}$, $w \in W_{n+1}, h \in H$ and $h N \notin \widetilde{W}_{n}$. This yields $x=w h n$ for some $n \in N$, and hence $w^{-1} x=h n$. But $w^{-1} x \in W_{n+1} W_{n+1} \subset W_{n}$, yielding $w^{-1} x N \in \widetilde{W}_{n}$. Hence, $h N=h n N=w^{-1} x N \in \widetilde{W}_{n}$, a contradiction.

Next we define

$$
Q_{n}=P_{1} \cap \cdots \cap P_{n} .
$$

Clearly, every $Q_{n}$ is a neighborhood of $e N$ in $\widetilde{G}$. We claim that $\left\{Q_{n}\right\}_{n=1}^{\infty}$ is an open base at $e N$ in $\widetilde{G}$. To prove this, let $Y$ be any neighborhood of $e$ in $G$. One has to find a $k$ such that $Q_{k} \subset \widetilde{Y}$.

First we choose a neighborhood $V$ of $e$ in $G$ such that $V^{2} \subset Y$. Then there are $m$ such that $\widetilde{W}_{m} \cap \widetilde{H} \subset \widetilde{V} \cap \widetilde{H}$ and $k>m$ such that $\varphi\left(U_{k}\right) \subset$ $\varphi\left(V \cap W_{m+1}\right)$. This implies that $U_{k} H \subset\left(V \cap W_{m+1}\right) H$, which, in turn, yields $U_{k} \widetilde{H} \subset\left(V \cap W_{m+1}\right) \widetilde{H}$.

Then we have

$$
\begin{aligned}
Q_{k} & \subset P_{k} \cap P_{m} \subset\left(U_{k} \widetilde{H}\right) \cap\left(\widetilde{G} \backslash \overline{W_{m+1}\left(\widetilde{H} \backslash \widetilde{W}_{m}\right)}\right) \\
& \subset\left(V \cap W_{m+1}\right) \widetilde{H} \cap\left(\widetilde{G} \backslash\left(\left(V \cap W_{m+1}\right)\left(\widetilde{H} \backslash \widetilde{W}_{m}\right)\right)\right) .
\end{aligned}
$$

Since

$$
\widetilde{G} \backslash\left(\left(V \cap W_{m+1}\right)\left(\widetilde{H} \backslash \widetilde{W}_{m}\right)\right)=\left(V \cap W_{m+1}\right)(\widetilde{G} \backslash \widetilde{H}) \cup\left(V \cap W_{m+1}\right) \widetilde{W}_{m},
$$

we get

$$
\begin{aligned}
Q_{k} & \subset\left(V \cap W_{m+1}\right) \widetilde{H} \cap\left(\left(V \cap W_{m+1}\right)(\widetilde{G} \backslash \widetilde{H}) \cup\left(V \cap W_{m+1}\right) \widetilde{W}_{m}\right) \\
& =\left(V \cap W_{m+1}\right) \widetilde{H} \cap\left(V \cap W_{m+1}\right) \widetilde{W}_{m}=\left(V \cap W_{m+1}\right)\left(\widetilde{H} \cap \widetilde{W}_{m}\right) \\
& \subset\left(V \cap W_{m+1}\right)(\widetilde{V} \cap \widetilde{H}) \subset V \widetilde{V}=\widetilde{V^{2}} \subset \widetilde{Y}
\end{aligned}
$$

as required.

Lemma 6.4. Let $G$ be a any topological group, $H$ a subgroup of $G$, and $N$ a compact subgroup of $H$. Suppose that $G / H$ and $H / N$ are both metrizable. Then so is $G / N$. Moreover, there exists a $G$-invariant metric on $G / N$.

Proof. By Lemma 6.3, $G / N$ has a countable open base at each point. By compactness of $N$, this implies that $G / N$ is metrizable by a $G$-invariant metric (see [38, Theorem 1]).

Lemma 6.5. Let $G$ be a locally compact group and $H$ its compact subgroup such that $G / H$ is metric. Then for each metrizable $H$-space $S$ the twisted product $G \times_{H} S$ is metrizable.

Proof. On $G \times_{H} S$ consider the $H$-action restricted from the $G$-action. We claim that $\frac{G \times H S}{H}$ is homeomorphic to $\frac{G / H \times S}{H}$, where $H$ acts on $G / H \times S$ by $h(x H, s)=(h x H, h s)$. 
Indeed, for any $[g, s] \in G \times_{H} S$ denote by $[g, s]_{H}$ its $H$-orbit. Analogously for any $(g H, s) \in G / H \times S$ denote by $(g H, s)_{H}$ its $H$-orbit. Then it is easy to see that the map

$$
\varphi: \frac{G \times{ }_{H} S}{H} \rightarrow \frac{G / H \times S}{H}, \quad \varphi\left([g, s]_{H}\right)=\left(g^{-1} H, s\right)_{H},
$$

is the desired homeomorphism.

Since $H$ is compact and $S$ is metrizable, so is $\frac{G / H \times S}{H}$. As $\frac{G \times H S}{H}$ is homeomorphic to $\frac{G / H \times S}{H}$, it is metrizable as well. According to Theorem 6.2, it remains to prove that each $H$-orbit in $G \times_{H} S$ is metrizable. We will prove that, in fact, each $G$-orbit in $G \times_{H} S$ is metrizable. Indeed, since $G \times_{H} S$ is a proper $G$-space, for each $x \in G \times{ }_{H} S$ the orbit $G(x)$ is homeomorphic to $G / G_{x}$ (see [42, Proposition 1.1.5]).

Thus we only have to prove that $G(x)=G / G_{x}$ is metrizable for any $x=[g, s] \in G \times_{H} S$. Observe that $G(x)=G(y)$, where $y=[e, s]$. It is clear that $G_{y}=H_{y}$, and so we have to show the metrizability of $G / H_{y}$. To this end, observe that $H / H_{y}$ is metric because it is homeomorphic to the $H$-orbit $H(y)$ of the metrizable $H$-space $S$. It remains to apply Lemma 6.4 with $N=H_{y}$.

Proof of Theorem 6.1. Since $X / G$ is paracompact, it follows from [2, Theorem 1.12] that $X$ is paracompact as well. Now, to prove that $X$ is metrizable, it suffices to show that it is locally metrizable (see e.g., [19, Ch. II, Theorem 4.1]).

According to Approximate Slice Theorem 2.2, each point of $X$ has a tubular neighborhood $G(S)$, where $S$ is an $H$-slice for some compact large subgroup $H \subset G$. By Lemma 2.3, $G(S)$ is $G$-homeomorphic to $G \times_{H} S$. Since $H$ is a large subgroup, $G / H$ is metrizable (see Section 2).

We claim that $S$ is metrizable. Indeed, each $H$-orbit $H(s)$ with $s \in S$ is metrizable because $H(s)$ is a subspace of $G(s)$, which is metrizable by hypothesis. On the other hand, $S / H \cong\left(G \times_{H} S\right) / G$, which is metrizable since $\left(G \times_{H} S\right) / G \cong G(S) / G$ is a subspace of the metrizable space $X / G$. Now Theorem 6.2 shows that $S$ is metrizable.

Hence, Lemma 6.5 implies that $G \times_{H} S$ is also metrizable. Thus $G(S)$, and hence $X$, is metrizable. This proves the first part of the theorem.

The second part follows from [17, Theorem B], where it is proved that a metrizable proper $G$-space with a paracompact orbit space admits a $G$ invariant metric.

7. Orbit spaces of arbitrary $G-\mathbf{A}(\mathbf{N}) \mathbf{E}$ spaces. In this section we shall strengthen Theorems 4.1 and 4.5 in two directions; it turns out that the assumptions of metrizability and properness of $X$ can be dropped. 
THEOREM 7.1. Let $G$ be a locally compact group and $X$ any $G$-ANE (respectively, any $G$-AE). Assume that $H$ is a closed (respectively, almost connected) normal subgroup of $G$ such that all the $H$-orbits in $X$ are metrizable. Then $X / H$ is a $G / H$-ANE (respectively, $a G / H$-AE).

Proof. Let $B \in G / H-\mathcal{M}$. Let $L$ be a closed $G / H$-invariant subset of $B$ and let $s: L \rightarrow X / H$ be a $G / H$-map. Define $A \subset L \times X$ to be the fiber product of $s$ and $t$, where $t: X \rightarrow X / H$ is the $H$-orbit map. Then $A$ is a $G$-invariant subspace of $L \times X$ endowed with the diagonal action of $G$, and we have $A / H=L$ (see [31, Ch. 4, Proposition 4.1]). Since the $H$-orbit of each $a=(l, x) \in A$ lies in the metrizable space $L \times H(x)$, we conclude that $H(a)$ is also metrizable. So, all $H$-orbits of the $G$-space $A$, as well as its $H$-orbit space $A / H=L$, are metrizable. By Theorem 6.1, $A$ is metrizable. Now applying Theorem 5.2 (respectively, Theorem 5.1) we get a $G$-space $Z \in G$-M with $Z / H$ a $G / H$-invariant neighborhood of $L$ in $B$ (respectively, with $Z / H=B$ ) such that $A$ is a closed $G$-invariant subspace of $Z$.

Let $\psi: A \rightarrow X$ be the restriction of the projection $L \times X \rightarrow X$. Since $X \in$ $G$-ANE (respectively, $X \in G$-AE), there exist a $G$-invariant neighborhood $U$ of $A$ in $Z$ (respectively, $U=Z$ ) and a $G$-extension $\alpha: U \rightarrow X$ of the $G$-map $\psi$. It is easy to see that the induced $G / H$-map $\beta: U / H \rightarrow X / H$ is the desired $G / H$-extension of $s$.

This theorem is the most general (in fact, final) result about preservation of equivariant extension properties by the orbit space functor. Its particular case for $H=G$ was proved in [14, Theorem 6.4]. The case of an almost connected group $G$ and a phase space $X$ with $X / G$ paracompact was handled in [10]. The very first orbit space theorem was established in [6] for the actions of compact metrizable groups on metrizable spaces. Recently, this result was widely applied in the study of the topology of the Banach-Mazur compacta (see [9], [11], [13]). Other applications can be found in [6], [18] and [45].

The following specific case of Theorem 7.1 when $H=G$ is worth singling out:

COROLlary 7.2. Let $G$ be a locally compact (respectively, an almost connected) group and $X$ any $G$-ANE (respectively, any $G$-AE). Assume that all the orbits in $X$ are metrizable. Then $X / G$ is an ANE (respectively, an $\mathrm{AE})$.

We conclude this section with the following finite-dimensional analogue of Theorem 7.1.

Given a nonnegative integer $n$, denote by $G-\mathcal{M}(n)$ the subclass of $G-\mathcal{M}$ that consists of all $G$-spaces $X \in G$-M with $\operatorname{dim} X / G \leq n$, where $\operatorname{dim}$ stands for the covering dimension. 
Recall that if $Y \in G-\operatorname{ANE}(X)$ for any $X \in G-\mathcal{M}(n), n \geq 0$, then we say that $Y$ is a $G-\operatorname{ANE}(n)$. Respectively, if $Y \in G-\operatorname{AE}(X)$ for any $X \in G-\mathcal{M}(n)$, $n \geq 0$, then we say that $Y$ is a $G-\operatorname{AE}(n)$.

TheOREM 7.3. Let $G$ be a locally compact group and $X$ any $G$-ANE $(n)$ (respectively, any $G-\mathrm{AE}(n)), n \geq 0$. Assume that $H$ is a closed (respectively, almost connected) normal subgroup of $G$ such that all the $H$-orbits in $X$ are metrizable. Then $X / H$ is a $G / H-\operatorname{ANE}(n)$ (respectively, $G / H-\operatorname{AE}(n)$ ).

The proof of this theorem is quite similar to the one of Theorem 7.1. The additional condition on the dimension of the orbit space holds because the relation $Z / H=B$ implies $Z / G=B /(G / H)$ (here we use the notation of the proof of Theorem 7.1).

REMARK 7.4. The metrizability condition for the $H$-orbits of $X$ in Theorems 7.1 and 7.3, as well as in Corollary 7.2, is evidently satisfied when $X$ is metrizable. Moreover, if $H$ is metrizable, then each $H$-orbit $H(x), x \in X$, is also metrizable. This follows from the fact that $H(x)$ is homeomorphic to the quotient space $H / H_{x}$, where $H_{x}=\{h \in H \mid h x=x\}$ (see [42, Proposition 1.1.5]), and metrizability of $H$ implies that of $H / H_{x}$ (see e.g. [40, Ch. I, $\S 1.23])$.

8. Appendix: Almost connected groups and maximal compact subgroups. In this section we shall establish some properties of almost connected groups and their maximal compact subgroups which we have used in Section 4.

Recall that a locally compact group $G$ is called almost connected if the space of connected components of $G$ is compact. Such a group has a maximal compact subgroup $K$, i.e., every compact subgroup of $G$ is conjugate to a subgroup of $K$ [1, Theorem A.5]. The corresponding classical theorem on Lie groups can be found in [30, Ch. XV, Theorem 3.1].

In what follows we shall use, without explicit reference, the well known fact that a locally compact subgroup of any topological group is a closed subset.

Proposition 8.1. Let $G$ be an almost connected group and $H$ a closed normal subgroup of $G$. Then $G / H$ is also almost connected.

Proof. Consider the following commutative diagram:

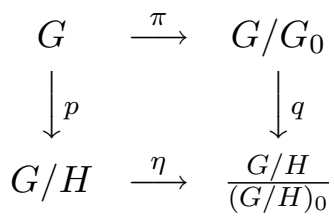


where $G_{0}$ and $(G / H)_{0}$ are the connected components of $G$ and $G / H$, respectively, and $\pi, p$ and $\eta$ are the natural homomorphisms.

The map $q$ is defined as follows:

$$
q\left(g G_{0}\right)=\langle g H\rangle
$$

where $\langle g H\rangle$ stands for the coset of $g H \in G / H$ with respect to the subgroup $(G / H)_{0} \subset G / H$. The map $q$ is well defined, because if $g_{0} \in G_{0}$ then

$$
q\left(g g_{0} G_{0}\right)=\left\langle g g_{0} H\right\rangle=\left\langle g H \cdot g_{0} H\right\rangle=\langle g H\rangle \cdot\left\langle g_{0} H\right\rangle .
$$

Observe that $g_{0} H \in p\left(G_{0}\right)$, and since $p\left(G_{0}\right)$ is connected, we infer that $p\left(G_{0}\right) \subset(G / H)_{0}$. Thus $g_{0} H \in(G / H)_{0}$, which yields $\langle g H\rangle \cdot\left\langle g_{0} H\right\rangle=\langle g H\rangle$.

Thus, $\frac{G / H}{(G / H)_{0}}$, being the continuous image of the compact group $G / G_{0}$, is itself compact.

Proposition 8.2. Let $G$ be any topological group and $H$ a normal subgroup of $G$. Then the connected component $H_{0}$ of $H$ is a normal subgroup of $G$.

Proof. Fix $g \in G$. Then $g H_{0} g^{-1} \subset g H g^{-1}=H$. Since $g H_{0} g^{-1}$ is homeomorphic to $H_{0}$, it is connected, and hence contained in $H_{0}$. Thus $g H_{0} g^{-1} \subset H_{0}$. Now replacing $g$ by $g^{-1}$ we get $g^{-1} H_{0} g \subset H_{0}$, which is equivalent to $H_{0} \subset g H_{0} g^{-1}$. Consequently, $g H_{0} g^{-1}=H_{0}$, as required.

Proposition 8.3. Let $G$ be a locally compact group and $H$ an almost connected normal subgroup of $G$ such that $G / H$ is compact. Then $G$ is also almost connected.

Proof. Let $G_{0}$ and $H_{0}$ be the connected components of $G$ and $H$, respectively. Clearly, $H_{0} \subset G_{0}$ and $H / H_{0}$ is compact. By Proposition 8.2, $H_{0}$ is a normal subgroup of $G$. Consider the natural homomorphism

$$
\pi: G / H_{0} \rightarrow \frac{G / H_{0}}{H / H_{0}}
$$

and observe that the groups $G / H$ and $\frac{G / H_{0}}{H / H_{0}}$ are topologically isomorphic. Since the fibers of $\pi$ are all homeomorphic to $H / H_{0}$, and hence are compact, we conclude that $G / H_{0}$ is compact (see [26, Theorem 3.7.24]).

Then $G / G_{0}$, being the continuous image of the compact group $G / H_{0}$ under the natural homomorphism $p: G / H_{0} \rightarrow G / G_{0}$ (remember that $H_{0} \subset$ $G_{0}$ ), is itself a compact group. Thus $G$ is almost connected, as required.

Proposition 8.4. Let $K$ be a maximal compact subgroup of $G$, and $N$ a compact normal subgroup of $G$ such that $N \subset K$. Then $K^{\prime}=K / N$ is a maximal compact subgroup of $G / N$.

Proof. Observe that $K^{\prime}$ is the image of $K$ under the natural homomorphism $p: G \rightarrow G / N$, so $K^{\prime}$ is a compact subgroup of $G / N$. Compactness of 
$N$ implies that $p$ is a perfect map. Since compactness is an inverse invariant of a perfect map (see [26, Theorem 3.7.24]) we infer that, for every compact subgroup $H^{\prime}$ of $G / N$, the inverse image $H=p^{-1}\left(H^{\prime}\right)$ is a compact subgroup of $G$.

Next, as $K$ is a maximal compact subgroup of $G$, there exists $g \in G$ such that $g \mathrm{Hg}^{-1} \subset K$. This yields $p\left(g H g^{-1}\right) \subset p(K)$. Since $p$ is a homomorphism,

$$
p\left(g H g^{-1}\right)=p(g) p(H) p(g)^{-1}=p(g) H^{\prime} p(g)^{-1} .
$$

Hence $p(g) H^{\prime} p(g)^{-1} \subset K^{\prime}$, as required.

THEOREM 8.5. Let $G$ be an almost connected group and $H$ an almost connected normal subgroup of $G$. Assume that $G / H$ is compact. Then $G=$ $K H$ for every maximal compact subgroup $K$ of $G$.

Proof. Case 1. Assume that, in addition, $H$ is connected. Let $K$ be a maximal compact subgroup of $G$. Since $G$ is almost connected, by a result of Glushkov [29, Theorem 8], there exists a compact normal subgroup $N \subset K$ such that $G / N$ is a Lie group. Consider the following commutative diagram:

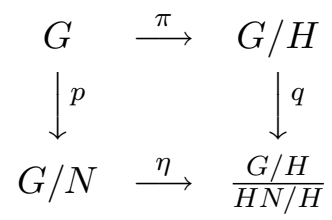

where $\pi, p$ and $q$ are the natural homomorphisms and $\eta$ is defined from the commutativity of the diagram.

Observe that in the case of Lie groups the assertion is proved in $[30$, p. 186, Theorem 3.7] and we are going to apply it to the Lie group $G / N$ and its closed connected normal subgroup $H N / N=p(H)$.

Indeed, the quotient group $\frac{G / N}{H N / N}$ is compact since it is topologically isomorphic to $\frac{G / H}{H N / H}$ (this is easy to check), which is just the image of the compact group $G / H$ under the continuous homomorphism $q$. Now applying [30, p. 186, Theorem 3.7] we get

$$
G / N=(H N / N) \cdot(K / N) .
$$

Here we have used the fact that $K / N$ is a maximal compact subgroup of $G / N$ (see Proposition 8.4). But since $N \subset K$ we see that $(H N / N) \cdot(K / N)=$ $H K / N$. Thus $G / N=H K / N$, yielding $G=H K$, as required.

Case 2. Assume that $H$ is an arbitrary almost connected closed normal subgroup of $G$. According to the previous case,

$$
G=G_{0} \cdot K
$$

where $G_{0}$ is the connected component of $G$. Let $H_{0}$ be the connected component of $H$. Clearly, $H_{0}$ is a normal closed subgroup of $G_{0}$. 
We are going to apply Case 1 to $G_{0}$ and $H_{0}$.

So, let us first show that $G_{0} / H_{0}$ is compact. Consider the natural continuous epimorphism $p: G \rightarrow G / H$. Since $p$ is constant on all the cosets $g H_{0}$, $g \in G$, it induces a continuous epimorphism $\pi: G / H_{0} \rightarrow G / H$. The fibers of $\pi$ are homeomorphic to its kernel, which is the quotient group $H / H_{0}$. Now, since $G / H$ and all the fibers of $\pi$ are compact, the group $G / H_{0}$ is also compact (see [26, Theorem 3.7.24]). Since $G_{0} / H_{0}$ is a closed subgroup of $G / H_{0}$, we conclude that $G_{0} / H_{0}$ is compact too.

Hence, Case 1 is applicable to the pair $\left(G_{0}, H_{0}\right)$, and so $G_{0}=L \cdot H_{0}$ for any maximal compact subgroup of $L$ of $G_{0}$. But observe that $L \subset g K^{-1}$ for some $g \in G$, so we get

$$
G_{0}=L \cdot H_{0} \subset\left(g K g^{-1}\right) \cdot H_{0} \subset\left(g K g^{-1}\right) \cdot H \subset g K H g^{-1} .
$$

Since $G_{0}$ is a normal subgroup, this is equivalent to the inclusion

$$
G_{0} \subset K H \text {. }
$$

Now, (8.1) and (8.3) imply that

$$
G=G_{0} K \subset K H K=K K H=K H,
$$

as required.

THEOREM 8.6. Let $G$ be an almost connected group, $H$ an almost connected normal subgroup of $G$, and $K$ a maximal compact subgroup of $G$. Then the image of $K$ under the natural homomorphism $\pi: G \rightarrow G / H$ is a maximal compact subgroup of $G / H$.

Proof. Case 1. First we assume that $H$ is connected. Let $M$ be any compact subgroup of $G / H$ and let $M^{\prime}=\pi^{-1}(M)$.

We claim that $M^{\prime}$ is almost connected. Indeed, denote by $M_{0}^{\prime}$ the connected component of $M^{\prime}$. Since $H$ is connected and $H \subset M^{\prime}$ we see that $H \subset M_{0}^{\prime}$. Hence, the natural homomorphism $p: M^{\prime} \rightarrow M^{\prime} / M_{0}^{\prime}$ factorizes through $M$ :

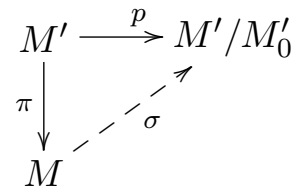

i.e., $p=\sigma \pi$, where $\sigma$ is a continuous homomorphism. Hence $M^{\prime} / M_{0}^{\prime}$ is the image of the compact group $M$ under the continuous homomorphism $\sigma$, and hence is compact. This shows that $M^{\prime}$ is almost connected.

Consequently, according to Theorem $8.5, M^{\prime}=L \cdot H$ for any maximal compact subgroup $L$ of $M^{\prime}$. Since $L \subset g K g^{-1}$ for some $g \in G$, we infer that

$$
M^{\prime} \subset\left(g K g^{-1}\right) \cdot H=g K \cdot H g^{-1} .
$$


This yields

$$
M=\pi\left(M^{\prime}\right) \subset \pi(g) \pi(K) \pi(g)^{-1},
$$

which means exactly that $\pi(K)$ is a maximal compact subgroup of $G / H$.

Case 2. Let $H$ be any almost connected group. Observe that, by Proposition 8.2, the connected component $H_{0}$ of $H$ is a normal subgroup of $G$. Consider the natural epimorphisms

$$
G \stackrel{\lambda}{\rightarrow} G / H_{0} \stackrel{\mu}{\rightarrow} G / H .
$$

According to Case $1, \lambda(K)$ is a maximal compact subgroup of $G / H_{0}$. Further, by Proposition 8.1, G/ $H_{0}$ is an almost connected group and $H / H_{0}$ is its compact normal subgroup. Now according to Proposition 8.4, $\mu(\lambda(K))$ is a maximal compact subgroup of $\frac{G / H_{0}}{H / H_{0}}$. It remains to observe that $G / H=\frac{G / H_{0}}{H / H_{0}}$ and $\pi(K)=\mu(\lambda(K))$.

Acknowledgements. The author would like to thank the referee whose thorough comments allowed improving the previous version of the text.

This research was partially supported by the grants \# CB-79536 from CONACYT (Mexico) and IN-102608 from PAPIIT (UNAM).

\section{References}

[1] H. Abels, Parallelizability of proper actions, global K-slices and maximal compact subgroups, Math. Ann. 212 (1974), 1-19.

[2] -, A universal proper G-space, Math. Z. 159 (1978), 143-158.

[3] N. Antonyan, S. Antonyan and L. Rodríguez-Medina, Linearization of proper group actions, Topology Appl. 156 (2009), 1946-1956.

[4] S. A. Antonyan, Retracts in categories of G-spaces, Izv. Akad. Nauk Armyan. SSR Ser. Mat. 15 (1980), 365-378 (in Russian); English transl.: Soviet J. Contemp. Math. Anal. 15 (1980), 30-43.

[5] -, Equivariant embeddings into G-AR's, Glas. Mat. 22 (42) (1987), 503-533.

[6] - Retraction properties of the orbit space, Mat. Sb. 137 (1988), 300-318 (in Russian); English transl.: Math. USSR-Sb. 65 (1990), 305-321.

[7] —, Existence of a slice for arbitrary compact transformation groups, Mat. Zametki 56 (1994), no. 5, 3-9 (in Russian); English transl.: Math. Notes 56 (1994), 1101-1104.

[8] - Preservation of $k$-connectedness and local $k$-connectedness by the symmetric $n$-th power functor, Moscow Univ. Math. Bull. 49 (1994), 22-25.

[9] - , The Banach-Mazur compacta are absolute retracts, Bull. Polish Acad. Sci. Math. 46 (1998), 113-119.

[10] - Extensorial properties of orbit spaces of proper group actions, Topology Appl. 98 (1999), 35-46.

[11] - , The topology of the Banach-Mazur compactum, Fund. Math. 166 (2000), 209232.

[12] —, Universal proper G-spaces, Topology Appl. 117 (2002), 23-43.

[13] - West's problem on equivariant hyperspaces and Banach-Mazur compacta, Trans. Amer. Math. Soc. 355 (2003), 3379-3404. 
[14] S. A. Antonyan, Orbit spaces and unions of equivariant absolute extensors, Topology Appl. 146-147 (2005), 289-315.

[15] —, Orbit spaces of proper equivariant absolute extensors, ibid. 153 (2005), 698-709.

[16] —, G-ANR's with homotopy trivial fixed point sets, Fund. Math. 197 (2007), 1-16.

[17] S. A. Antonyan and S. de Neymet, Invariant pseudometrics on Palais proper $G$ spaces, Acta Math. Hungar. 98 (2003), 41-51.

[18] V. N. Basmanov, Functors and equivariant absolute retracts, Moscow Univ. Math. Bull. 49 (1994), no. 4, 24-26.

[19] C. Bessaga and A. Pełczyński, Selected Topics in Infinite-Dimensional Topology, PWN-Polish Sci. Publ., Warszawa, 1975.

[20] N. P. Bhatia and G. P. Szegö, Stability Theory of Dynamical Systems, Springer, Berlin, 1970.

[21] G. Bredon, Introduction to Compact Transformation Groups, Academic Press, New York, 1972.

[22] T. tom Dieck, Transformation Groups, de Gruyter, Berlin, 1987.

[23] J. Dugundji, An extension of Tietze's theorem, Pacific J. Math. 1 (1951), 353-367.

[24] E. Elfving, The G-homotopy type of proper locally linear $G$-manifolds, Ann. Acad. Sci. Fenn. Math. Dissertationes 108 (1996).

[25] —, The G-homotopy type of proper locally linear G-manifolds. II, Manuscripta Math. 105 (2001), 235-251.

[26] R. Engelking, General Topology, PWN-Polish Sci. Publ., Warszawa, 1977.

[27] A. Feragen, Equivariant embedding of metrizable G-spaces in linear G-spaces, Proc. Amer. Math. Soc. 136 (2008), 2985-2995.

[28] V. V. Filippov, On weight characteristics of spaces with a compact group action, Math. Notes 25 (1979), 939-947.

[29] V. M. Glushkov, The structure of locally compact groups and Hilbert's fifth problem, Amer. Math. Soc. Transl. 15 (1960), 59-93.

[30] G. Hochschild, The Structure of Lie Groups, Holden-Day, San Francisco, 1965.

[31] D. Husemoller, Fiber Bundles, Springer, 1994.

[32] E. Hewitt and K. Ross, Abstract Harmonic Analysis, Vol. I, Springer, 1963.

[33] S. Illman, Every proper smooth action of a Lie group is equivalent to a real analytic action: a contribution to Hilbert's fifth problem, in: Prospects in Topology, F. Quinn (ed.), Ann. of Math. Stud. 138, Princeton Univ. Press, 1995, 189-220.

[34] I. M. James and G. B. Segal, On equivariant homotopy theory, in: Lecture Notes in Math. 788, Springer, 1980, 316-330.

[35] M. Kankaanrinta, Proper smooth G-manifolds have G-invariant Riemannian metrics, Topology Appl. 153 (2005), 610-619.

[36] V. L. Klee, A note on topological properties of normed linear spaces, Proc. Amer. Math. Soc. 7 (1956), 673-674.

[37] J. L. Koszul, Lectures on Groups of Transformations, Tata Inst. Fund. Res., Bombay, 1965.

[38] L. Kristensen, Invariant metrics in coset spaces, Math. Scand. 6 (1958), 33-36.

[39] E. Michael, Some extension theorems for continuous functions, Pacific J. Math. 3 (1953), 789-806.

[40] D. Montgomery and L. Zippin, Topological Transformation Groups, Krieger, Huntington, 1974.

[41] R. Palais, The classification of G-spaces, Mem. Amer. Math. Soc. 36 (1960).

[42] - On the existence of slices for actions of non-compact Lie groups, Ann. of Math. 73 (1961), 295-323. 
[43] B. A. Pasynkov, On spaces with a compact group of transformations, Soviet Math. Dokl. 17 (1976), 1522-1526.

[44] E. V. Ščepin, On Tychonoff manifolds, ibid. 20 (1979), 511-515.

[45] T. F. Žuraev, On covariant functors of finite degree that preserve A(N)R-spaces, C. R. Acad. Bulgare Sci. 43 (1990), no. 9, 5-8 (in Russian).

Departamento de Matemáticas

Facultad de Ciencias

Universidad Nacional Autónoma de México

04510 México D.F., Mexico

E-mail: antonyan@unam.mx

Received 29 May 2008;

in revised form 3 August 2009 